Supporting Information for

\title{
Protein-Peptide Binding Energetics under Crowded Conditions
}

\author{
Samantha S. Stadmiller, ${ }^{1}$ Jhoan S. Aguilar, ${ }^{1}$ Stuart Parnham, ${ }^{2}$ Gary J. Pielak ${ }^{1,2,3,4^{*}}$ \\ ${ }^{1}$ Department of Chemistry, University of North Carolina, Chapel Hill, North Carolina 27599 \\ ${ }^{2}$ Department of Biochemistry and Biophysics, University of North Carolina, Chapel Hill, North Carolina \\ 27599 \\ ${ }^{3}$ Lineberger Comprehensive Cancer Center, University of North Carolina, Chapel Hill, North Carolina \\ 27599 \\ ${ }^{4}$ Integrative Program for Biological and Genome Sciences, University of North Carolina, Chapel Hill, North \\ Carolina 27599
}

${ }^{*}$ Correspondence to: Gary J. Pielak, Department of Chemistry, University of North Carolina, Chapel Hill, North Carolina 27599, United States. E-mail: gary_pielak@unc.edu 


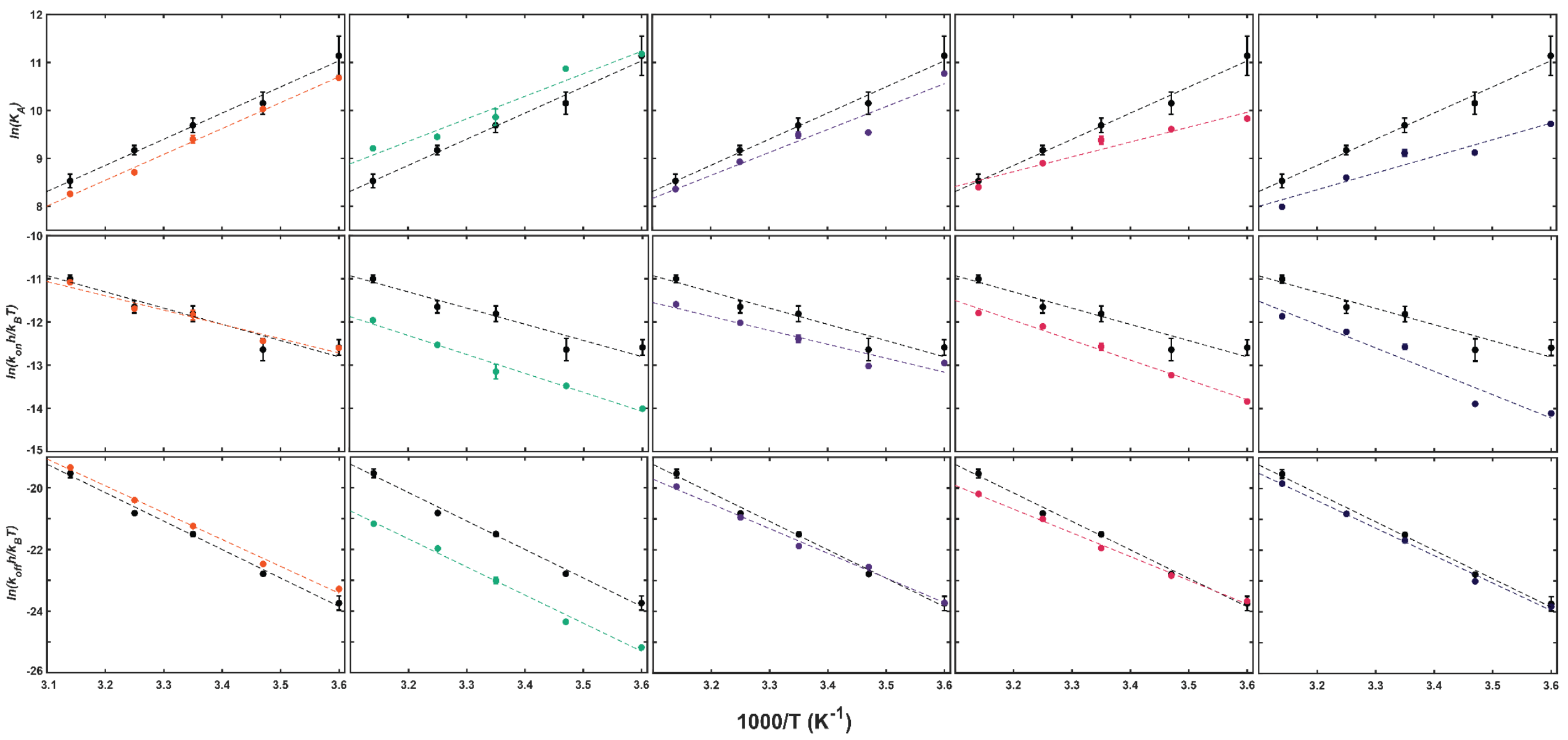

Figure S1. Eyring (middle and bottom) and van't Hoff (top) plots for SH3-PepS4 binding in the presence of cosolutes. Data in buffer are shown in black with error bars representing standard errors of the mean from replicate measurements at each temperature. ${ }^{1}$ For cosolutes, measurements were made in triplicate at $298.15 \mathrm{~K}$ error bars on these points represent the standard error of the mean from replicates. Cosolute experiments at other temperatures were performed once. Analysis of uncertainty for enthalpy and entropy is described in the Materials and Methods. 
A

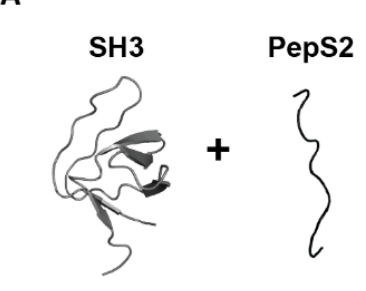

Peps2

YRAVPBPLPBRE
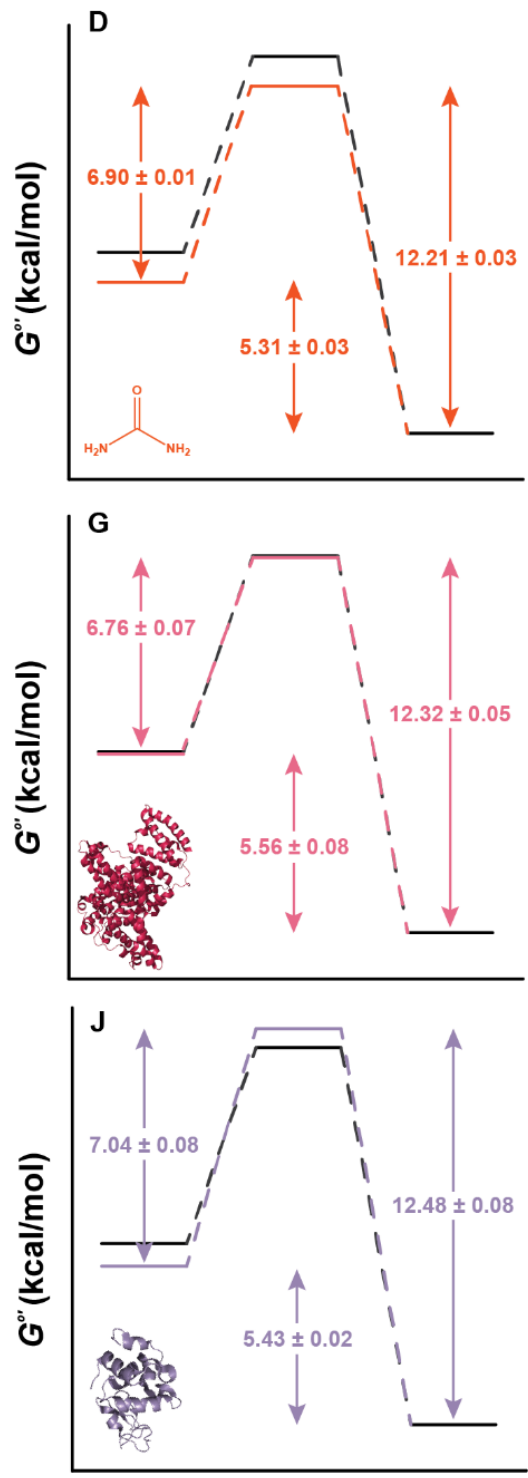
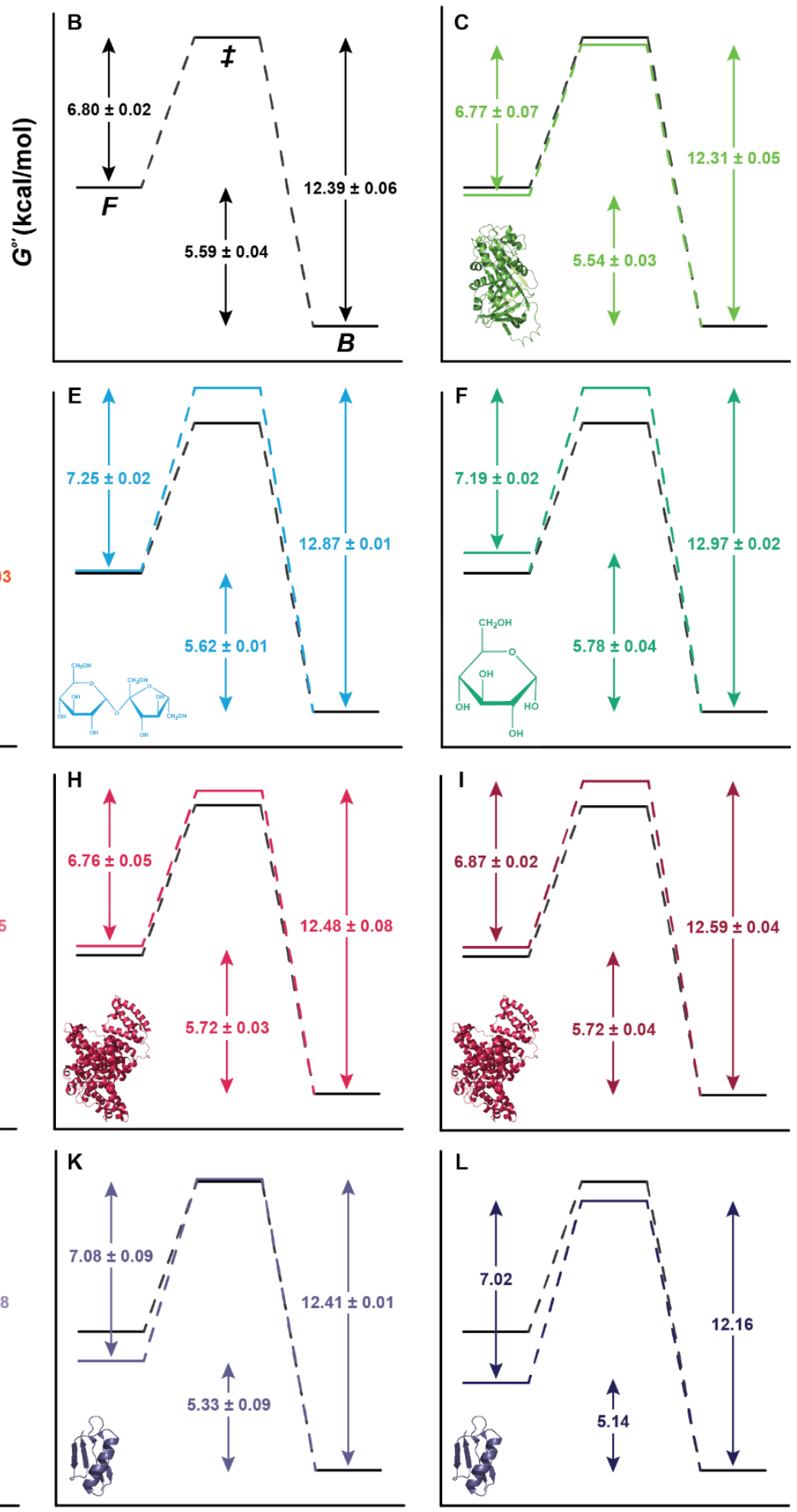

\section{Reaction Coordinate}

Figure S2. Free energy diagrams for SH3-PepS2 binding in cosolutes at $318.15 \mathrm{~K}$. Reaction and peptide sequence (A). SH3-PepS2 binding in buffer [B ( $F$, free; ₹, transition state; B, bound)]. Effect of 50 $\mathrm{g} / \mathrm{L}$ ovalbumin (C), $50 \mathrm{~g} / \mathrm{L}$ urea (D), $300 \mathrm{~g} / \mathrm{L}$ sucrose (E), $300 \mathrm{~g} / \mathrm{L}$ glucose (F), $50 \mathrm{~g} / \mathrm{L}$ BSA (G), $100 \mathrm{~g} / \mathrm{L}$ BSA (H), $200 \mathrm{~g} / \mathrm{L} \mathrm{BSA}(\mathrm{I}), 50 \mathrm{~g} / \mathrm{L}$ lysozyme (J), $50 \mathrm{~g} / \mathrm{L}$ GB1 (K), and $100 \mathrm{~g} / \mathrm{L}$ GB1 (L). 

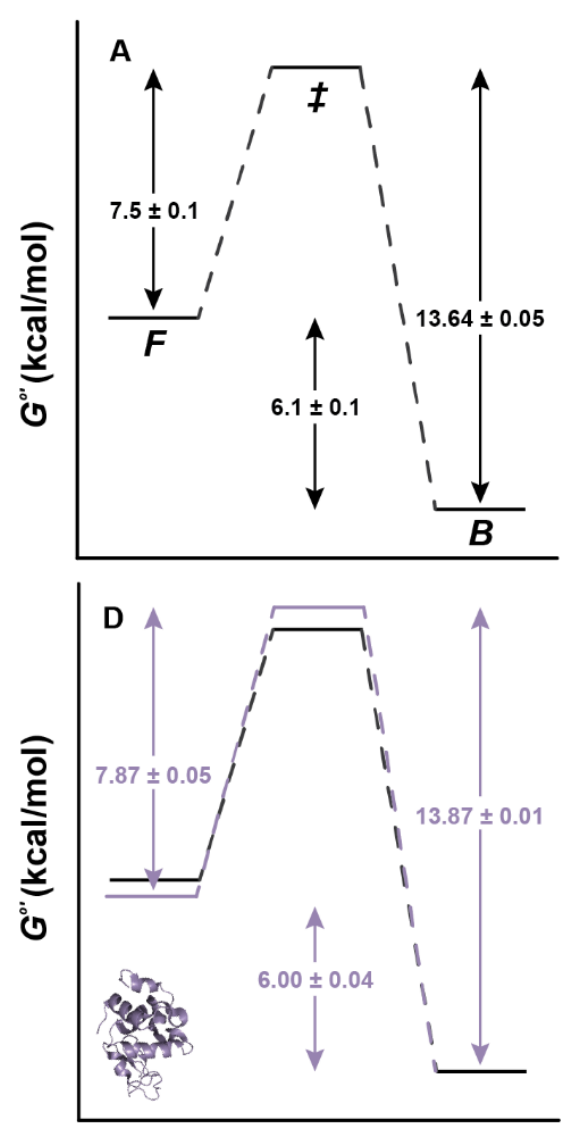
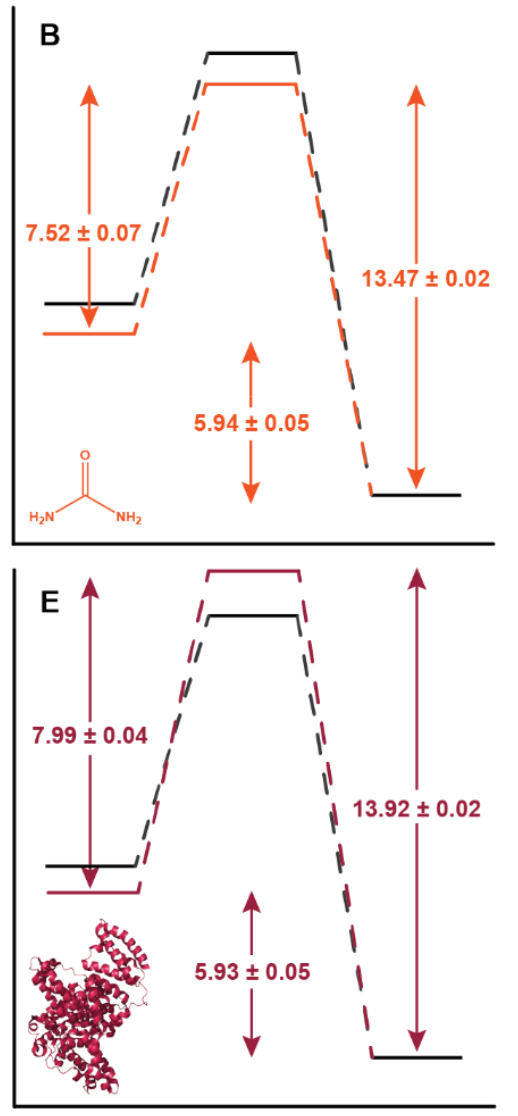

\section{Reaction Coordinate}
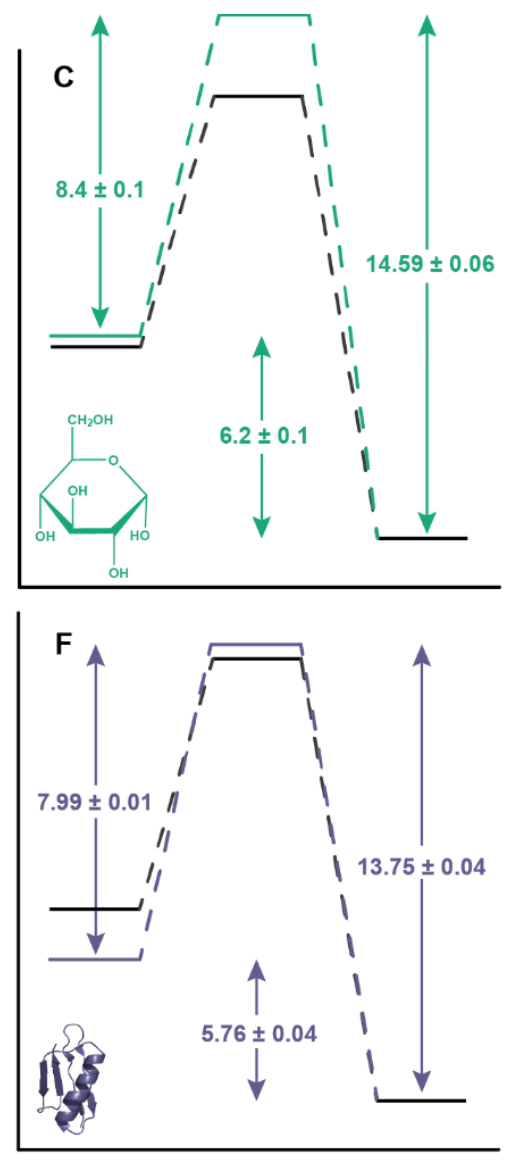

Figure S3. Free energy diagrams for SH3-PepS4 binding in cosolutes at 298.15 K. Free energy parameters for SH3-PepS4 binding in buffer (A). Effect of cosolutes on free energy diagrams for SH3PepS4 binding in $50 \mathrm{~g} / \mathrm{L}$ urea (B), $300 \mathrm{~g} / \mathrm{L}$ glucose (C), $50 \mathrm{~g} / \mathrm{L}$ lysozyme (D), $200 \mathrm{~g} / \mathrm{L}$ BSA (E), and $50 \mathrm{~g} / \mathrm{L}$ GB1 (F). 


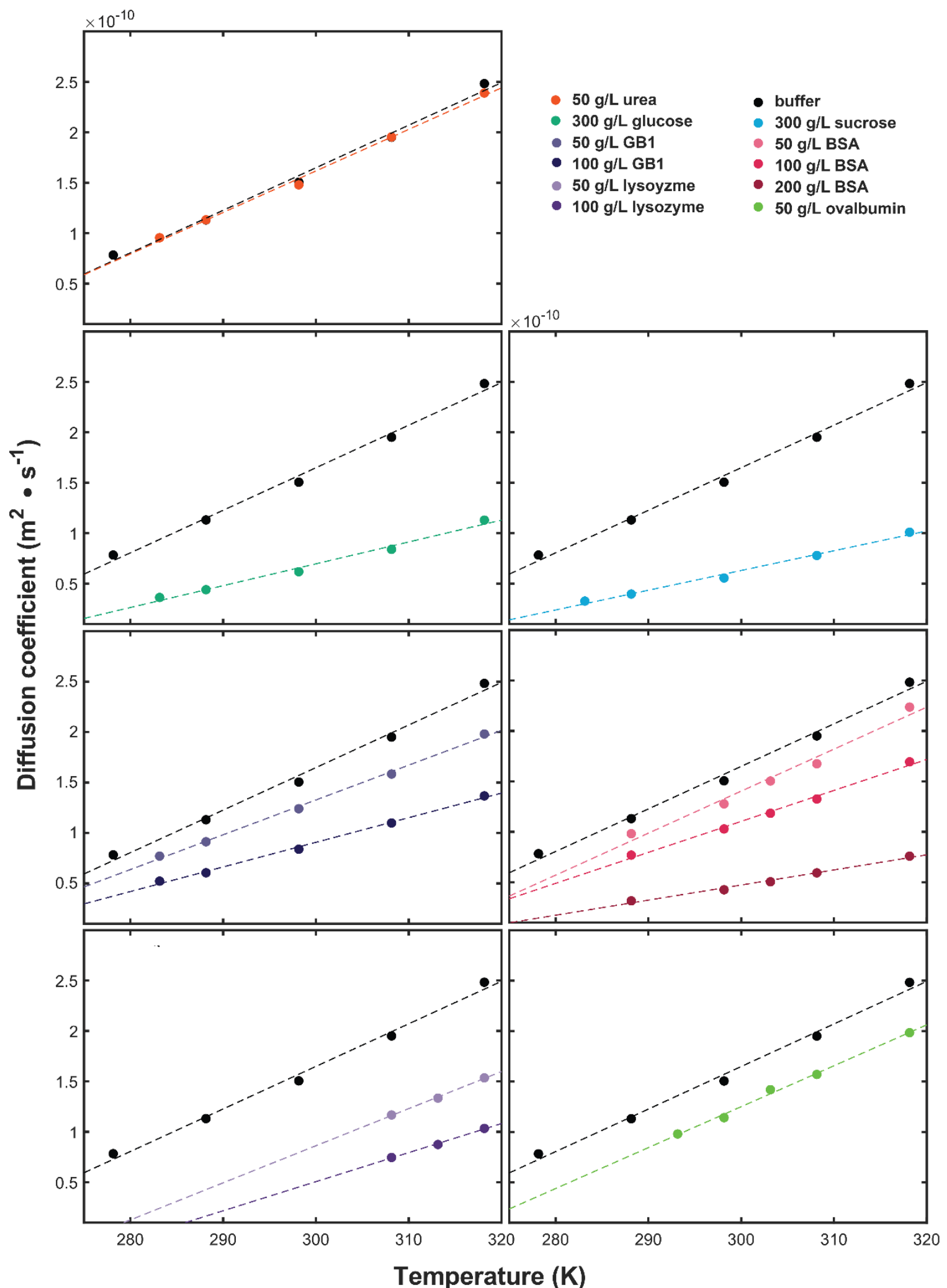

Figure S4. Translational diffusion of $\mathrm{SH} 3$ as a function of temperature and cosolute. Results from ${ }^{19} \mathrm{~F}$ DOSY experiments are plotted against absolute temperature. 


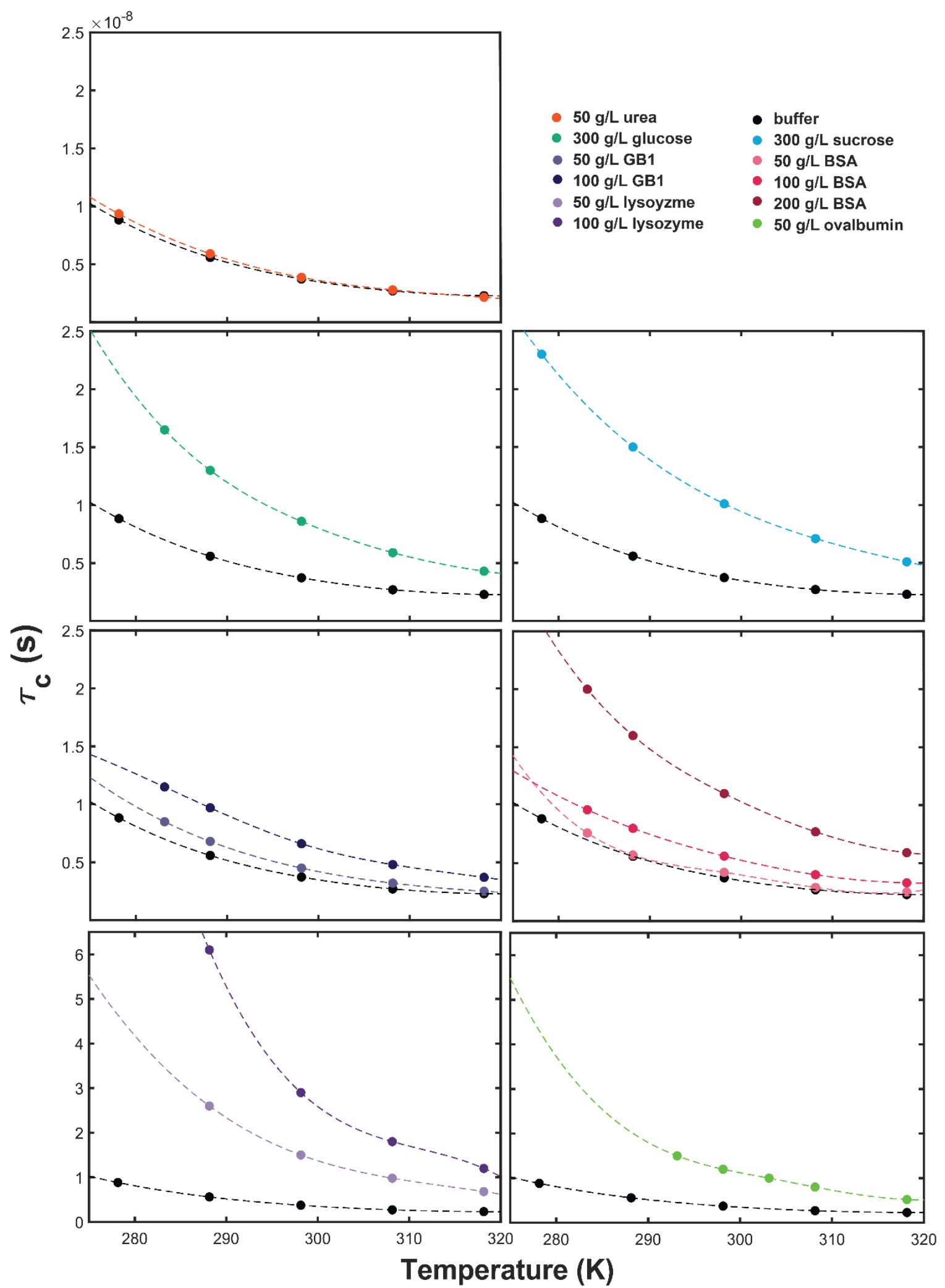

Figure S5. Rotational correlation times of $\mathrm{SH}_{3}$ as a function of temperature and cosolute. $\mathrm{R}_{1}$ and $\mathrm{R}_{2}$ measurements with further fitting of the Model-Free formalism are plotted against temperature. Dashed lines are of no theoretical significance and are provided to aid visualization. 


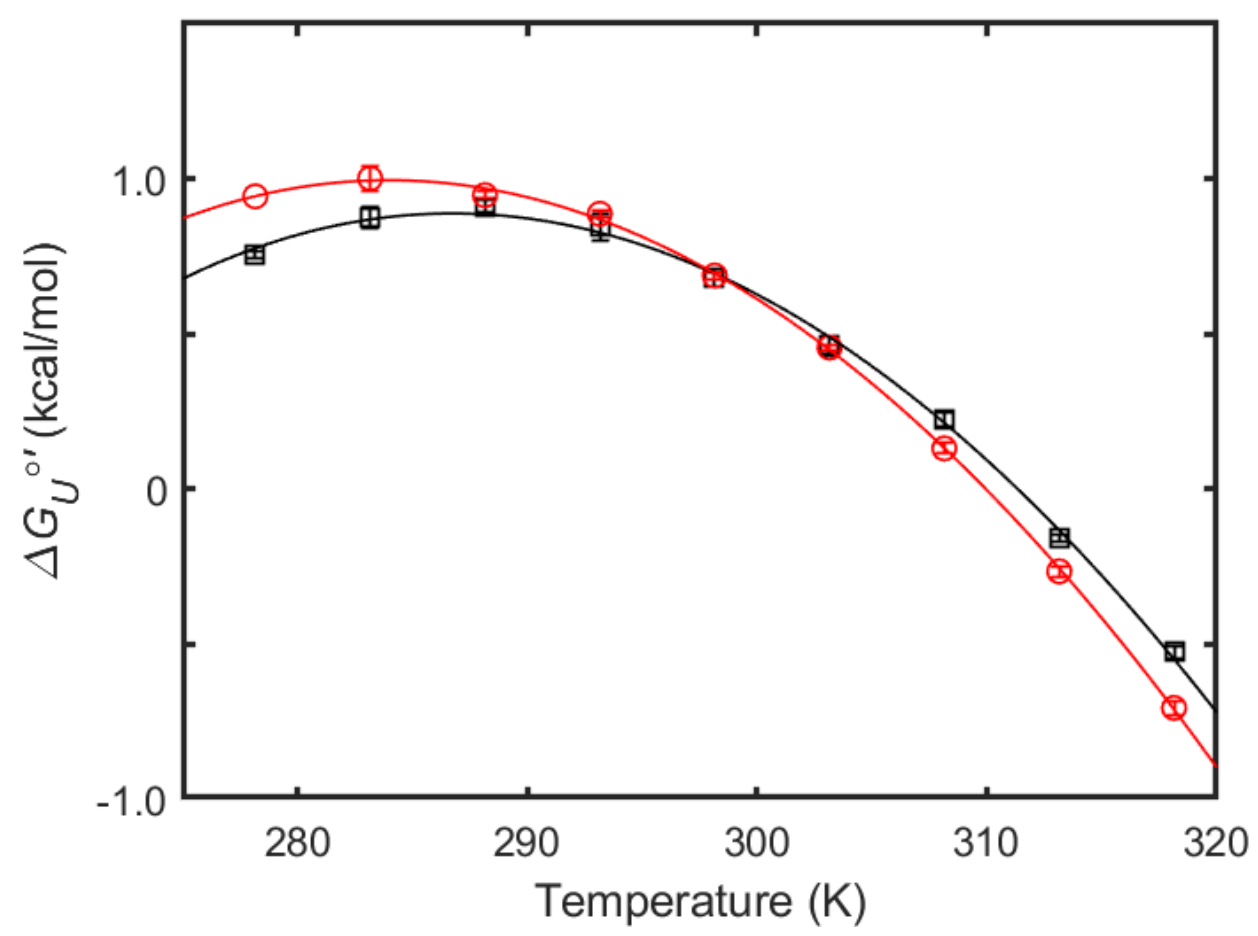

Figure S6. Stability curve of $0.3 \mathrm{mM}$ wild-type SH3 in buffer (black) and in $50 \mathrm{~g} / \mathrm{L} \mathrm{GB1}$ (red). Error bars represent standard errors of the mean from triplicate measurements. Temperature dependence was fit using the integrated Gibbs-Helmholtz equation. ${ }^{2-4}$ 
Table S1. Parameters used for acquiring ${ }^{19} \mathrm{~F}$ DOSY experiments of $\mathrm{SH} 3$ in cosolutes

\begin{tabular}{|c|c|c|c|}
\hline Cosolute & Temperature (K) & $\Delta / \mathrm{d} 20(\mathrm{~ms})$ & ర / p30 (ms) \\
\hline \multirow{5}{*}{ buffer } & 278.15 & 60 & 3.0 \\
\hline & 288.15 & 60 & 2.5 \\
\hline & 298.15 & 60 & 2.25 \\
\hline & 308.15 & 60 & 2.0 \\
\hline & 318.15 & 60 & 2.0 \\
\hline \multirow{5}{*}{$50 \mathrm{~g} / \mathrm{L}$ urea } & 283.15 & 60 & 3.0 \\
\hline & 288.15 & 60 & 2.5 \\
\hline & 298.15 & 60 & 2.25 \\
\hline & 308.15 & 60 & 2.0 \\
\hline & 318.15 & 60 & 2.0 \\
\hline \multirow{5}{*}{$\begin{array}{c}300 \mathrm{~g} / \mathrm{L} \text { glucose and } \\
300 \mathrm{~g} / \mathrm{L} \text { sucrose }\end{array}$} & 283.15 & 210 & 2.25 \\
\hline & 288.15 & 180 & 2.25 \\
\hline & 298.15 & 150 & 2.25 \\
\hline & 308.15 & 150 & 2.0 \\
\hline & 318.15 & 150 & 1.75 \\
\hline \multirow{5}{*}{$50 \mathrm{~g} / \mathrm{L}$ ovalbumin } & 293.15 & 60 & 2.5 \\
\hline & 298.15 & 60 & 2.5 \\
\hline & 303.15 & 60 & 2.5 \\
\hline & 308.15 & 60 & 2.25 \\
\hline & 318.15 & 60 & 2.25 \\
\hline \multirow{5}{*}{$50 \mathrm{~g} / \mathrm{L} \mathrm{BSA}$} & 288.15 & 60 & 2.75 \\
\hline & 298.15 & 60 & 2.5 \\
\hline & 303.15 & 60 & 2.5 \\
\hline & 308.15 & 60 & 2.25 \\
\hline & 318.15 & 60 & 2.0 \\
\hline \multirow{5}{*}{$100 \mathrm{~g} / \mathrm{L} \mathrm{BSA}$} & 288.15 & 60 & 2.75 \\
\hline & 298.15 & 60 & 2.5 \\
\hline & 303.15 & 60 & 2.75 \\
\hline & 308.15 & 60 & 2.5 \\
\hline & 318.15 & 60 & 2.25 \\
\hline \multirow{5}{*}{$200 \mathrm{~g} / \mathrm{L} \mathrm{BSA}$} & 288.15 & 150 & 2.75 \\
\hline & 298.15 & 120 & 2.75 \\
\hline & 303.15 & 120 & 2.75 \\
\hline & 308.15 & 120 & 2.5 \\
\hline & 318.15 & 120 & 2.25 \\
\hline \multirow{5}{*}{$50 \mathrm{~g} / \mathrm{L}$ GB1 } & 283.15 & 60 & 3.0 \\
\hline & 288.15 & 60 & 2.75 \\
\hline & 298.15 & 60 & 2.5 \\
\hline & 308.15 & 60 & 2.25 \\
\hline & 318.15 & 60 & 2.0 \\
\hline \multirow{5}{*}{100 g/L GB1 } & 283.15 & 90 & 3.0 \\
\hline & 288.15 & 90 & 2.75 \\
\hline & 298.15 & 90 & 2.5 \\
\hline & 308.15 & 90 & 2.25 \\
\hline & 318.15 & 90 & 2.0 \\
\hline \multirow{3}{*}{$50 \mathrm{~g} / \mathrm{L}$ lysozyme } & 308.15 & 60 & 2.5 \\
\hline & 313.15 & 60 & 2.5 \\
\hline & 318.15 & 60 & 2.5 \\
\hline \multirow{3}{*}{$100 \mathrm{~g} / \mathrm{L}$ lysozyme } & 308.15 & 60 & 2.75 \\
\hline & 313.15 & 60 & 2.5 \\
\hline & 318.15 & 90 & 2.5 \\
\hline
\end{tabular}


Table S2. Parameters for BSA and lysozyme in the Vogel-Fulcher equation.

\begin{tabular}{ccc} 
Parameter (units) & BSA & Lysozyme \\
\hline$M_{p}(\mathrm{amu})$ & 66,000 & 14,320 \\
$\alpha\left(\mathrm{kg} / \mathrm{m}^{3}\right)$ & $3.67 \times 10^{6}$ & $7.96 \times 10^{5}$ \\
$v\left(\mathrm{~m}^{3} / \mathrm{kg}\right)$ & $1.42 \times 10^{-3}$ & $2.59 \times 10^{-3}$ \\
$B_{P}$ & $8.39 \times 10^{5}$ & $2.64 \times 10^{4}$ \\
$D_{P}\left(\mathrm{~K}^{-1}\right)$ & 649 & 42 \\
$\Delta E_{P}(\mathrm{~kJ} / \mathrm{mol})$ & $5.37 \times 10^{5}$ & $3.97 \times 10^{4}$
\end{tabular}

Table S3. Apparent relative viscosities calculated from diffusion measurements at $298.15 \mathrm{~K}$

\begin{tabular}{|c|c|c|c|}
\hline Cosolute & $\eta_{c} / \eta_{b}$ (literature) & $\begin{array}{c}\eta_{c} / \eta_{b} \\
\text { (translational } \\
\text { diffusion) }\end{array}$ & $\begin{array}{c}\eta_{c} / \eta_{b} \\
\text { (rotational diffusion) }\end{array}$ \\
\hline $50 \mathrm{~g} / \mathrm{L}$ urea & $1.2^{\mathrm{a}}$ & 1.1 & 1.2 \\
\hline $300 \mathrm{~g} / \mathrm{L}$ sucrose & $3.0^{\mathrm{a}}$ & 2.9 & 2.7 \\
\hline $300 \mathrm{~g} / \mathrm{L}$ glucose & $2.8^{\mathrm{a}}$ & 2.6 & 3.2 \\
\hline 50 g/L lysozyme & 1.2 & $1.8^{\mathrm{b}}$ & 3.2 \\
\hline $100 \mathrm{~g} / \mathrm{L}$ lysozyme & 1.4 & $2.8^{\mathrm{b}}$ & 6.0 \\
\hline $50 \mathrm{~g} / \mathrm{L} \mathrm{BSA}$ & 1.4 & 1.3 & 1.3 \\
\hline $100 \mathrm{~g} / \mathrm{L} \mathrm{BSA}$ & 2.0 & 1.6 & 1.8 \\
\hline $200 \mathrm{~g} / \mathrm{L} \mathrm{BSA}$ & 5.0 & 3.8 & 3.5 \\
\hline $50 \mathrm{~g} / \mathrm{L}$ ovalbumin & $1.4^{\mathrm{c}}$ & 1.4 & 3.2 \\
\hline $50 \mathrm{~g} / \mathrm{L}$ GB1 & $\mathrm{n} / \mathrm{a}$ & 1.3 & 1.4 \\
\hline $100 \mathrm{~g} / \mathrm{L}$ GB1 & $\mathrm{n} / \mathrm{a}$ & 1.9 & 2.1 \\
\hline
\end{tabular}


Table S4. Eyring parameters for SH3-PepS4 binding at $298.15 \mathrm{~K}$

\begin{tabular}{|c|c|c|c|c|}
\hline Cosolute & Reaction & $\begin{array}{c}\Delta G_{298 \mathrm{~K}}^{\circ / \neq} \\
(\mathrm{kcal} / \mathrm{mol})\end{array}$ & $\Delta H^{\circ \prime}(\mathrm{kcal} / \mathrm{mol})$ & $\begin{array}{c}T \Delta S_{298 K}^{\circ / \neq} \\
\text { (kcal/mol) }\end{array}$ \\
\hline \multirow[t]{2}{*}{ buffer } & $F \rightarrow \ddagger$ & $7.5 \pm 0.1$ & $7.3 \pm 0.9$ & $0.2 \pm 0.9$ \\
\hline & $B \rightarrow \neq$ & $13.6 \pm 0.1$ & $18 \pm 1$ & $5.5 \pm 0.9$ \\
\hline \multirow[t]{2}{*}{$50 \mathrm{~g} / \mathrm{L}$ urea } & $F \rightarrow \ddagger$ & $\begin{array}{l}7.5 \pm 0.1 \\
6.9 \pm 0.1^{a}\end{array}$ & $\begin{array}{c}6.6 \pm 0.5 \\
6.6 \pm 0.5^{a}\end{array}$ & $\begin{array}{c}-1 \pm 2 \\
-0.4 \pm 0.5^{a}\end{array}$ \\
\hline & $B \rightarrow \ddagger$ & $\begin{array}{c}13.5 \pm 0.1 \\
12.5 \pm 0.1^{a}\end{array}$ & $\begin{array}{c}17.5 \pm 0.7 \\
16.9 \pm 0.7^{a}\end{array}$ & $\begin{array}{c}4.9 \pm 0.7 \\
5 \pm 1^{a}\end{array}$ \\
\hline \multirow[t]{2}{*}{$300 \mathrm{~g} / \mathrm{L}$ glucose } & $F \rightarrow \neq$ & $\begin{array}{c}8.4 \pm 0.1 \\
7.2 \pm 0.1^{a}\end{array}$ & $\begin{array}{c}9 \pm 1 \\
8 \pm 1^{a}\end{array}$ & $\begin{array}{c}1 \pm 1 \\
1 \pm 1^{a}\end{array}$ \\
\hline & $B \rightarrow \neq$ & $\begin{array}{c}14.6 \pm 0.1 \\
13.0 \pm 0.1^{a}\end{array}$ & $\begin{array}{c}18 \pm 2 \\
17 \pm 2^{a}\end{array}$ & $\begin{array}{c}4 \pm 2 \\
4 \pm 2^{a}\end{array}$ \\
\hline \multirow[t]{2}{*}{$50 \mathrm{~g} / \mathrm{L}$ lysozyme } & $F \rightarrow \ddagger$ & $\begin{array}{c}7.9 \pm 0.1 \\
7.3 \pm 0.1^{a}\end{array}$ & $\begin{array}{c}6.5 \pm 0.3 \\
6.3 \pm 0.4^{a}\end{array}$ & $\begin{array}{c}-0 . \overline{8} \pm 0.4 \\
-0.9 \pm 0.4^{\mathrm{a}}\end{array}$ \\
\hline & $B \rightarrow \ddagger$ & $\begin{array}{c}13.9 \pm 0.1 \\
12.9 \pm 0.1^{\mathrm{a}}\end{array}$ & $\begin{array}{c}16.1 \pm 0.5 \\
15.8 \pm 0.5^{a}\end{array}$ & $\begin{array}{c}3.2 \pm 0.5 \\
3.0 \pm 0.5^{a}\end{array}$ \\
\hline \multirow[t]{2}{*}{$200 \mathrm{~g} / \mathrm{L} \mathrm{BSA}$} & $F \rightarrow \ddagger$ & $\begin{array}{c}8.0 \pm 0.1 \\
6.5 \pm 0.1^{a}\end{array}$ & $\begin{array}{c}9.2 \pm 0.3 \\
8.0 \pm 0.3^{a}\end{array}$ & $\begin{array}{c}1.7 \pm 0.3 \\
1.5 \pm 0.3^{a}\end{array}$ \\
\hline & $B \rightarrow \ddagger$ & $\begin{array}{c}13.9 \pm 0.1 \\
12.1 \pm 0.1^{\mathrm{a}}\end{array}$ & $\begin{array}{c}15.5 \pm 0.6 \\
14.2 \pm 0.6^{a}\end{array}$ & $\begin{array}{c}2.5 \pm 0.6 \\
2.2 \pm 0.6^{a}\end{array}$ \\
\hline \multirow[t]{2}{*}{$50 \mathrm{~g} / \mathrm{L} \mathrm{GB1}$} & $F \rightarrow \ddagger$ & $8.0 \pm 0.1$ & $10.9 \pm 0.1$ & $3.3 \pm 0.1$ \\
\hline & $B \rightarrow \ddagger$ & $13.8 \pm 0.1$ & $17.9 \pm 0.8$ & $5.0 \pm 0.8$ \\
\hline
\end{tabular}

${ }^{\mathrm{a} C a l c u l a t e d}$ using viscosity-adjusted rate constant

Table S5. Equilibrium thermodynamic parameters for SH3-PepS4 binding

\begin{tabular}{|c|c|c|c|}
\hline Cosolute & $\Delta G_{D, 298 K}^{\circ \prime}(\mathrm{kcal} / \mathrm{mol})$ & $\Delta H_{D}^{\circ \prime}(\mathrm{kcal} / \mathrm{mol})$ & $T \Delta S_{D, 298 K}^{\circ \prime}(\mathrm{kcal} / \mathrm{mol})$ \\
\hline buffer & $6.1 \pm 0.1$ & $11 \pm 2$ & $5 \pm 2$ \\
\hline $50 \mathrm{~g} / \mathrm{L}$ urea & $5.9 \pm 0.1$ & $10.8 \pm 0.9$ & $5.3 \pm 0.8$ \\
\hline $300 \mathrm{~g} / \mathrm{L}$ glucose & $6.2 \pm 0.1$ & $9 \pm 2$ & $3 \pm 2$ \\
\hline $50 \mathrm{~g} / \mathrm{L}$ lysozyme & $6.0 \pm 0.1$ & $9.6 \pm 0.7$ & $4.0 \pm 0.7$ \\
\hline $200 \mathrm{~g} / \mathrm{L} \mathrm{BSA}$ & $5.9 \pm 0.1$ & $6.2 \pm 0.8$ & $0.8 \pm 0.8$ \\
\hline $50 \mathrm{~g} / \mathrm{L}$ GB1 & $5.8 \pm 0.1$ & $7.0 \pm 0.7$ & $1.7 \pm 0.7$ \\
\hline
\end{tabular}


Table S6. Temperature-dependence of relaxation rates, rotational correlation times, and translational diffusion of $\mathrm{SH} 3$ in various cosolutes

\begin{tabular}{|c|c|c|c|c|c|}
\hline Cosolute & $\begin{array}{c}\text { Temperature } \\
(\mathrm{K})\end{array}$ & $\mathbf{R}_{1}\left(\mathbf{s}^{-1}\right)$ & $\mathbf{R}_{\mathbf{2}}\left(\mathbf{s}^{-1}\right)$ & $\tau_{c}(n s)$ & $\begin{array}{c}D_{\text {T }}\left(x \mathbf{1 0}^{-10} \mathrm{~m}^{2}\right. \\
\left.\mathrm{s}^{-1}\right) \\
\end{array}$ \\
\hline \multirow{5}{*}{ buffer } & 278.15 & $2.48 \pm 0.03$ & $68 \pm 3$ & $8.8 \pm 0.4$ & 0.78 \\
\hline & 288.15 & $2.69 \pm 0.02$ & $43 \pm 1$ & $5.6 \pm 0.1$ & 1.1 \\
\hline & 298.15 & $2.62 \pm 0.04$ & $29 \pm 1$ & $3.7 \pm 0.1$ & 1.5 \\
\hline & 308.15 & $2.66 \pm 0.03$ & $22 \pm 1$ & $2.7 \pm 0.1$ & 2.0 \\
\hline & 318.15 & $2.22 \pm 0.05$ & $18 \pm 1$ & $2.3 \pm 0.2$ & 2.5 \\
\hline \multirow{6}{*}{$50 \mathrm{~g} / \mathrm{L}$ urea } & 278.15 & $2.43 \pm 0.03$ & $72 \pm 2$ & $9.3 \pm 0.2$ & $\overline{---}$ \\
\hline & 283.15 & -- & -- & -- & 0.96 \\
\hline & 288.15 & $2.60 \pm 0.02$ & $45 \pm 1$ & $5.9 \pm 0.1$ & 1.1 \\
\hline & 298.15 & $2.64 \pm 0.04$ & $30 \pm 1$ & $3.9 \pm 0.1$ & 1.5 \\
\hline & 308.15 & $2.46 \pm 0.01$ & $22 \pm 1$ & $2.8 \pm 0.2$ & 2.0 \\
\hline & 318.15 & $2.24 \pm 0.03$ & $17 \pm 1$ & $2.2 \pm 0.2$ & 2.4 \\
\hline \multirow{6}{*}{$\begin{array}{l}300 \mathrm{~g} / \mathrm{L} \\
\text { sucrose }\end{array}$} & 278.15 & $1.09 \pm 0.05$ & $170 \pm 10$ & $23 \pm 1$ & -- \\
\hline & 283.15 & --- & --- & --- & 0.33 \\
\hline & 288.15 & $1.49 \pm 0.01$ & $110 \pm 10$ & $15 \pm 1$ & 0.40 \\
\hline & 298.15 & $1.91 \pm 0.01$ & $74 \pm 1$ & $10.1 \pm 0.2$ & 0.56 \\
\hline & 308.15 & $2.24 \pm 0.01$ & $52 \pm 1$ & $7.1 \pm 0.1$ & 0.78 \\
\hline & 318.15 & $2.44 \pm 0.01$ & $38 \pm 1$ & $5.1 \pm 0.1$ & 1.0 \\
\hline \multirow{5}{*}{$\begin{array}{l}300 \mathrm{~g} / \mathrm{L} \\
\text { glucose }\end{array}$} & 283.15 & $1.44 \pm 0.02$ & $121 \pm 1$ & $16.5 \pm 0.1$ & 0.36 \\
\hline & 288.15 & $1.68 \pm 0.01$ & $95 \pm 1$ & $13.0 \pm 0.1$ & 0.44 \\
\hline & 298.15 & $2.09 \pm 0.01$ & $63 \pm 1$ & $8.6 \pm 0.1$ & 0.62 \\
\hline & 308.15 & $2.39 \pm 0.01$ & $44 \pm 1$ & $5.9 \pm 0.1$ & 0.84 \\
\hline & 318.15 & $2.52 \pm 0.02$ & $33 \pm 1$ & $4.3 \pm 0.1$ & 1.1 \\
\hline \multirow{5}{*}{$\begin{array}{c}50 \mathrm{~g} / \mathrm{L} \\
\text { Iysozyme }\end{array}$} & 288.15 & $1.50 \pm 0.02$ & $210 \pm 10$ & $26 \pm 1$ & 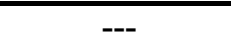 \\
\hline & 298.15 & $1.78 \pm 0.01$ & $110 \pm 10$ & $15 \pm 1$ & --- \\
\hline & 308.15 & $1.94 \pm 0.01$ & $72 \pm 1$ & $9.8 \pm 0.1$ & 1.2 \\
\hline & 313.15 & --- & --- & --- & 1.3 \\
\hline & 318.15 & $2.1 \pm 0.2$ & $49 \pm 1$ & $6.8 \pm 0.2$ & 1.5 \\
\hline \multirow{6}{*}{$\begin{array}{c}100 \mathrm{~g} / \mathrm{L} \\
\text { lysozyme }\end{array}$} & 278.15 & $0.74 \pm 0.02$ & -- & -- & --- \\
\hline & 288.15 & $0.94 \pm 0.01$ & $550 \pm 10$ & $61 \pm 1$ & --- \\
\hline & 298.15 & $1.36 \pm 0.02$ & $240 \pm 10$ & $29 \pm 1$ & --- \\
\hline & 308.15 & $1.59 \pm 0.01$ & $140 \pm 10$ & $18 \pm 1$ & 0.75 \\
\hline & 313.15 & -- & --- & -- & 0.87 \\
\hline & 318.15 & $1.78 \pm 0.01$ & $88 \pm 1$ & $12 \pm 1$ & 1.0 \\
\hline \multirow{6}{*}{$50 \mathrm{~g} / \mathrm{L} \mathrm{BSA}$} & 283.15 & $2.49 \pm 0.01$ & $58 \pm 1$ & $7.6 \pm 0.1$ & $\overline{---}$ \\
\hline & 288.15 & $2.59 \pm 0.02$ & $43 \pm 1$ & $5.7 \pm 0.1$ & 0.98 \\
\hline & 298.15 & $2.57 \pm 0.02$ & $32 \pm 1$ & $4.2 \pm 0.1$ & 1.3 \\
\hline & 303.15 & -- & -- & -- & 1.5 \\
\hline & 308.15 & $2.49 \pm 0.02$ & $23 \pm 1$ & $2.9 \pm 0.2$ & 1.7 \\
\hline & 318.15 & $2.19 \pm 0.02$ & $19 \pm 1$ & $2.5 \pm 0.1$ & 2.2 \\
\hline \multirow{6}{*}{$100 \mathrm{~g} / \mathrm{L} \mathrm{BSA}$} & 283.15 & $2.32 \pm 0.01$ & $73 \pm 1$ & $9.6 \pm 0.1$ & --- \\
\hline & 288.15 & $2.43 \pm 0.02$ & $61 \pm 1$ & $8.0 \pm 0.1$ & 0.77 \\
\hline & 298.15 & $2.46 \pm 0.02$ & $42 \pm 1$ & $5.6 \pm 0.1$ & 1.0 \\
\hline & 303.15 & --- & --- & --- & 1.2 \\
\hline & 308.15 & $2.40 \pm 0.02$ & $30 \pm 1$ & $4.0 \pm 0.2$ & 1.3 \\
\hline & 318.15 & $2.27 \pm 0.02$ & $25 \pm 1$ & $3.3 \pm 0.2$ & 1.7 \\
\hline \multirow{4}{*}{$200 \mathrm{~g} / \mathrm{L} \mathrm{BSA}$} & 283.15 & $1.76 \pm 0.01$ & $160 \pm 10$ & $20 \pm 1$ & $\overline{---}$ \\
\hline & 288.15 & $1.92 \pm 0.01$ & $130 \pm 10$ & $16 \pm 1$ & 0.32 \\
\hline & 298.15 & $2.13 \pm 0.01$ & $84 \pm 1$ & $11 \pm 1$ & 0.43 \\
\hline & 303.15 & -- & -- & -- & 0.50 \\
\hline
\end{tabular}




\begin{tabular}{cccccc} 
& 308.15 & $2.21 \pm 0.01$ & $57 \pm 1$ & $7.7 \pm 0.1$ & 0.59 \\
& 318.15 & $2.19 \pm 0.05$ & $43 \pm 1$ & $5.9 \pm 0.2$ & 0.76 \\
\hline \multirow{3}{*}{$50 \mathrm{~g} / \mathrm{L}$} & 293.15 & $2.38 \pm 0.01$ & $130 \pm 10$ & $15 \pm 1$ & 0.98 \\
ovalbumin & 298.15 & $2.39 \pm 0.01$ & $100 \pm 10$ & $12 \pm 1$ & 1.1 \\
& 303.15 & $2.39 \pm 0.01$ & $77 \pm 1$ & $10.0 \pm 0.1$ & 1.4 \\
& 308.15 & $2.34 \pm 0.01$ & $60 \pm 1$ & $8.0 \pm 0.1$ & 1.6 \\
& 318.15 & $2.23 \pm 0.01$ & $38 \pm 1$ & $5.2 \pm 0.1$ & 2.0 \\
\hline $50 \mathrm{~g} / \mathrm{L} \mathrm{GB1}$ & 283.15 & $2.37 \pm 0.01$ & $64 \pm 1$ & $8.5 \pm 0.1$ & 0.77 \\
& 288.15 & $2.48 \pm 0.01$ & $51 \pm 1$ & $6.8 \pm 0.1$ & 0.91 \\
& 298.15 & $2.55 \pm 0.01$ & $34 \pm 1$ & $4.5 \pm 0.1$ & 1.3 \\
& 308.15 & $2.46 \pm 0.03$ & $25 \pm 1$ & $3.2 \pm 0.2$ & 1.6 \\
$100 \mathrm{~g} / \mathrm{L}$ GB1 & 318.15 & $2.33 \pm 0.02$ & $20 \pm 1$ & $2.5 \pm 0.2$ & 2.0 \\
& 283.15 & $1.93 \pm 0.01$ & $86 \pm 1$ & $11.5 \pm 0.1$ & 0.52 \\
& 288.15 & $2.10 \pm 0.01$ & $72 \pm 1$ & $9.7 \pm 0.1$ & 0.61 \\
& 298.15 & $2.32 \pm 0.01$ & $49 \pm 1$ & $6.6 \pm 0.1$ & 0.84 \\
& 308.15 & $2.41 \pm 0.01$ & $36 \pm 1$ & $4.8 \pm 0.1$ & 1.1 \\
& 318.15 & $2.36 \pm 0.01$ & $28 \pm 1$ & $3.7 \pm 0.1$ & 1.4
\end{tabular}

\section{Citations}

(1) Stadmiller, S. S.; Aguilar, J. S.; Waudby, C. A.; Pielak, G. J., Biophys. J. 2020, 2537-2548.

(2) Becktel, W. J.; Schellman, J. A., Biopolymers 1987, 26, 1859-1877.

(3) Senske, M.; Smith, A. E.; Pielak, G. J., Angew. Chem., Int. Ed. 2016, 55, 3586-3589.

(4) Smith, A. E.; Zhou, L. Z.; Gorensek, A. H.; Senske, M.; Pielak, G. J., Proc. Natl. Acad. Sci. USA 2016, 113, 1725-1730.

(5) Concentrative Properties of Aqueous Solutions: Density, Refractive Index, Freezing Point Depression, and Viscosity. In CRC Handbook of Chemistry and Physics, 54 ed.; Weast, R. C., Ed. Chemical Rubber Publishing Company: Cleveland, Ohio, 1973.

(6) Monkos, K., Int. J. Biol. Macromol. 1996, 18, 61-68. 